\title{
Reflets
}

Revue ontaroise d'intervention sociale et communautaire

\section{Conviction de la différence}

synthèse d'une recherche sur la famille francophone en Ontario

\section{Christiane Bernier}

Volume 3, numéro 2, automne 1997

Visibles et Partenaires : Pratiques et recherches féministes

URI : https://id.erudit.org/iderudit/026174ar

DOI : https://doi.org/10.7202/026174ar

Aller au sommaire du numéro

Éditeur(s)

Reflets : Revue ontaroise d'intervention sociale et communautaire

ISSN

1203-4576 (imprimé)

1712-8498 (numérique)

Découvrir la revue

Citer cet article

Bernier, C. (1997). Conviction de la différence : synthèse d'une recherche sur la famille francophone en Ontario. Reflets, 3(2), 87-105.

https://doi.org/10.7202/026174ar
Résumé de l'article

Synthèse d'une recherche à trois volets sur la famille franco-ontarienne, ce texte présente les principaux résultats qu'ont révélé une comparaison entre familles francophones et familles anglophones en Ontario. Le premier volet établit un profil statistique des familles des deux groupes linguistiques. Le deuxième volet tente d'en mesurer la cohésion familiale et le troisième présente, à travers une analyse du discours, les éléments sur lesquels repose la constructionsymbolique de l'ethos de la famille franco-ontarienne.
Tous droits réservés (C) Reflets : Revue ontaroise d'intervention sociale et communautaire, 1997
Ce document est protégé par la loi sur le droit d'auteur. L'utilisation des services d’Érudit (y compris la reproduction) est assujettie à sa politique d'utilisation que vous pouvez consulter en ligne.

https://apropos.erudit.org/fr/usagers/politique-dutilisation/ 


\section{Conviction de la différence : synthèse $d$ 'une recherche sur la famille francophone en Ontario}

Synthèse d'une recherche à trois volets sur la famille franco-ontarienne, ce texte présente les principaux résultats qu'ont révélé une comparaison entre familles francophones et familles anglophones en Ontario. Le premier volet établit un profil statistique des familles des deux groupes linguistiques. Le deuxième volet tente d'en mesurer la cohésion familiale et le troisième présente, à travers une analyse du discours, les éléments sur lesquels repose la construction symbolique de l'ethos de la famille franco-ontarienne.

\section{Christiane Bernier}

Professeure, Département de sociologie, Université Laurentienne

\section{Mise en contexte}

En Ontario, depuis une dizaine d'années, les chercheures féministes francophones s'interrogent sur les liens entre identité de femme et identité culturelle (ou ethnique). Divers colloques ont été organisés et plusieurs écrits ont été publiés à cet égard. Une des pistes théoriques fécondes qui y fut développée fait état du rôle important de productrice d'ethnicité assumé par les femmes francophones en milieu minoritaire, tant au sein de leur famille que de leur communauté (McKee-Allain 1989). L'intérêt de cette approche est - et ce, malgré les diverses critiques que l'on peut émettre quant à la trop grande restriction de la vision que l'on y déploie (Bernier 1994) — qu'elle est la seule à avoir lié aux 
«Suivant le

mouvement, il est apparu pertinent de vérifier si... la famille franco-ontarienne correspond à la symbolique que l'on entretient constamment à son sujet en milieu minoritaire.» problématiques de l'identité de femme et de l'identité de minoritaire celle de la famille francophone.

Peu de recherches sociologiques, en effet, ont porté comme tel sur la famille francophone en milieu minoritaire ${ }^{1}$. Jusqu' à tout récemment, on l'avait presque toujours abordée d'un point de vue historique et socio-politique en faisant le creuset (avec la religion) de la reproduction du groupe minoritaire (Choquette 1977; Breton 1983; Bernard 1990; Juteau et Séguin-Kimpton 1993; Carrière 1993; Gervais 1993). En l'identifiant comme l'instrument de la victoire de la survie des francophones, on a toujours ainsi, et avec vigueur, revendiqué sa spécificité, sa différence, ses qualités particulières, contre la famille anglo-dominante.

L'Année internationale de la famille (1994), en créant un vaste espace réflexif sur tous les aspects liés à la famille a permis, non seulement de faire le point sur l'état des familles, au Canada comme ailleurs, mais a instigué des recherches sur la perception qu'ont les gens de la famille en général et de leur famille en particulier. Suivant le mouvement, il est apparu pertinent de vérifier si - et dans quelle mesure si tel est le cas - la famille franco-ontarienne correspond à la symbolique que l'on entretient constamment à son sujet en milieu minoritaire. Ainsi, on croit encore en effet que ses liens sont à ce point "tricotés serrés", que même les transformations profondes des dernières décennies ne peuvent l'affecter autant que les autres familles (Herry et Lévesque 1994).

Discours de la spécificité assurément, dont on pourrait dire qu'il est une sorte d'éthique de la conviction sans cesse reproduite. De façon caricaturale, on peut voir que cette conviction repose sur trois énoncés de base. La famille franco-ontarienne serait différente :

1. parce qu'il y aurait plus de cohésion dans cette famille que dans la famille anglo-ontarienne, c'est-à-dire que l'on croit qu'elle entretient des relations plus suivies avec ses différents membres, voire qu'elle est plus chaleureuse;

2. parce qu'elle serait plus nombreuse et conséquemment plus étendue;

3. et ceci encore plus au Nord qu'ailleurs en Ontario, puisque c'est là que l'on trouve la plus grande proportion de personnes de langue maternelle française. 
Sur quoi repose ce discours de la spécificité? Quel en est le fondement? Peut-on, à partir de diverses analyses, vérifier les éléments de cette construction symbolique? C'est cette question de base qui a servi d'objectif général à la recherche. Cette dernière s'est développée en trois étapes successives, cernant la problématique de diverses façons.

\section{Familles en Ontario: les indicateurs objectifs ${ }^{2}$}

Le premier volet de la recherche a voulu voir si, en établissant un profil statistique comparatif entre la famille francophone et la famille anglophone en Ontario, on arriverait à identifier, à partir de certains indicateurs objectifs - structure familiale, statut marital, nombre d'enfants, types et secteurs d'occupation, logement, profil d'éducation et revenus - des secteurs ou des lieux sociaux qui rendraient manifeste cette particularité des francophones. Cette première comparaison a été établie à partir d'analyses réalisées sur les données provenant du fichier de microdonnées à grande diffusion sur les familles (échantillon provincial) tiré du recensement canadien de 1991.

Les résultats de cette première étude ont démontré que, de façon générale, il y a effectivement certaines disparités entre les personnes vivant dans des familles francophones et les autres familles en Ontario, mais elles sont beaucoup moins prononcées que la conviction que l'on semble en avoir. Les quelques différences dont on peut faire état se situent dans les domaines de la structure de la famille, de l'éducation, des revenus familiaux et des types et secteurs d'occupation. Voici quelques exemples de données sur trois des points soulevés ${ }^{3}$.

Structure de la famille

Autour de $80 \%$ des familles en Ontario sont constituées de personnes légalement mariées. Ce mode familial reste donc la norme encore aujourd'hui. Avec certaines différences pourtant: les hommes ont plus tendance à se marier que les femmes et les 
"...du côté des femmes: les francophones forment une plus grande proportion que les non-francophones à n'avoir pas fait d'études secondaires... et sont moins bien représentées au niveau du postsecondaire.» non-francophones plus que les francophones. En fait, c'est chez les femmes francophones que l'on retrouve, proportionnellement, le moins de personnes mariées $(80,9 \%)$ alors qu'à l'opposé, c'est parmi les hommes anglophones que l'on en voit la proportion la plus élevée (90,3 \%). Il n'est pas étonnant, dès lors, de constater que les francophones choisissent un peu plus que les anglophones de vivre en union de fait. Il faut signaler aussi qu'il y a une plus grande proportion de familles monoparentales chez les francophones, ceci pouvant être en partie lié au fait qu'il y a davantage de pères francophones chefs de famille monoparentale.

\section{Éducation}

Les tendances générales démontrent que, comme de par le passé, les francophones sont moins instruits que les non-francophones. Ainsi, les hommes n'atteignent pas le secondaire dans une proportion plus grande et poursuivent moins des études postsecondaires non universitaires ou universitaires de premier cycle que les personnes vivant dans des familles non francophones. Par contre, et ceci est nouveau, au niveau des études de deuxième cycle, le pourcentage d'anglophones et de francophones se répartit également désormais.

Le modèle est à peine différent du côté des femmes: les francophones forment une plus grande proportion que les nonfrancophones à n'avoir pas fait d'études secondaires (quoiqu'elles en aient fait davantage que leurs homologues masculins) et sont moins bien représentées au niveau du postsecondaire. Mais, contrairement aux hommes francophones, et cela aussi est nouveau, elles surpassent légèrement les femmes non francophones au niveau des études supérieures ( $2^{\mathrm{e}}$ cycle).

\section{Revenus familiaux}

Il y a une différence entre le revenu annuel moyen d'une famille non francophone (56 $150 \$$ ) et le revenu annuel moyen d'une famille francophone (51 593 \$), en Ontario, à la défaveur de celle-ci. Cet écart moyen est de 4557 \$. Bien que relativement peu élevé, l'écart entre hommes francophones et hommes anglophones ou allophones vivant en famille - les personnes vivant 
"...les femmes vivant dans une famille font moins de la moitié des revenus annuels de leur partenaire.» «...il n'est plus vrai de dire, ... que la famille francoontarienne est plus nombreuse...» seules ont été exclues de l'échantillon - est statistiquement significatif, de même que celui entre femmes francophones et femmes non francophones. Mais c'est, à n'en pas douter, entre les femmes et les hommes que cet écart est le plus marquant. En effet, autant chez les francophones que chez les anglophones, les femmes vivant dans une famille font moins de la moitié des revenus annuels de leur partenaire.

\section{Pour le Nord}

Chacune des analyses a aussi comparé les francophones du Nord à ceux des autres régions, pour vérifier la troisième sous-hypothèse.

Certaines disparités ont été relevées pour le Nord, notamment en ce qui a trait à l'éducation, les francophones étant moins instruits que partout ailleurs en Ontario; à la structure d'emploi, surtout chez les hommes francophones pour qui le chômage est plus prégnant; et aux rapports familiaux où l'homme francophone du Nord se trouve en plus grande proportion seul soutien (financier) de famille.

\section{Conclusion du premier volet}

Bref, dans l'ensemble, les résultats de cette première étape de l'analyse nous font voir que les distinctions entre familles francophones et familles anglophones ne sont pas si prononcées que nous aurions pu le croire et que, souvent, nous trouvons davantage d'écarts entre les régions ou entre hommes et femmes qu'entre francophones et anglophones en Ontario. Ces distinctions ne sont, à tout le moins, pas suffisantes pour expliquer que perdure une telle conviction de la différence.

Ainsi, il n'est plus vrai de dire, par exemple, comme nous pouvions le faire encore à la génération précédente, que la famille franco-ontarienne est plus nombreuse - tout au moins pour les familles ayant actuellement des enfants à la maison - ni que cela s'avère plus au Nord qu'ailleurs en Ontario; par contre, le type d'informations recueillies dans cette analyse ne permettait pas d'établir une comparaison en ce qui a trait à la taille de la famille étendue chez les personnes de chacun des deux groupes linguistiques. 
La spécificité des familles francophones en Ontario, peut-on penser alors, serait plus à chercher du côté de la vision qu'en entretiennent les Franco-Ontariens et les Franco-Ontariennes. Si leur conviction de cette spécificité demeure, c'est qu'elle doit reposer sur d'autres sortes d'éléments, par exemple sur des éléments qui seraient liés à la dynamique des relations intrafamiliales: unité au sein de la famille, partage de temps en commun, communication de tout type entre les membres, sentiment d'appartenance, etc.

Y aurait-il donc réellement plus de cohésion, plus de solidarité, des liens plus étroits entre les membres des familles francophones qu'entre ceux des familles anglo-ontariennes? Cette question peut s'appréhender de deux façons : soit en vérifiant s'il y a, empiriquement, un fondement à l'assertion de plus grande cohésion familiale chez les francophones, auquel cas on se donnera des indicateurs de mesure de cette solidarité; soit en l'abordant en termes de représentations sociales et en tentant de voir, à partir du discours que les personnes elles-mêmes produisent sur leur famille, comment et sur quoi s'articulent les éléments de cette construction symbolique. Les deux axes de recherche ont été développés.

\section{Cohésion familiale: fréquence des communications et type d'échanges ${ }^{4}$}

Le deuxième volet a porté sur l'analyse de la cohésion dans les familles francophones et anglophones par le biais des solidarités familiales, suivant en cela les tendances théoriques récentes en recherche de la famille (Pitrou 1992). Une des façons de mesurer cette cohésion, selon Pitrou (1992), est de déterminer la fréquence des communications entre les différents membres d'une même famille, d'identifier avec qui les personnes disent avoir des liens privilégiés et de voir s'il y a - et de quel type — des liens d'échange et d'entraide entre les membres. 


\section{L'enquête}

Nous avons donc effectué une enquête auprès d'un échantillon (stratifié, par grappes) de mères ${ }^{5}$ francophones et non francophones de la ville de Sudbury $(n=302)$. Cette ville du Nord, dont le bilinguisme est connu, a été choisie tout particulièrement pour répondre à la sous-hypothèse mentionnée plus haut. La collecte de données s'est effectuée à partir d'un questionnaire d'entrevue structurée; le questionnaire comprenait vingt et une questions, dont onze portaient sur le statut socio-économique et sur la situation de famille. L'enquête s'est déroulée en décembre 1994 et en janvier 1995.

\section{Les éléments de mesure de la solidarité}

Le concept de solidarité familiale a été opérationnalisé en mesurant les facteurs suivants :

1. l'existence de relations privilégiées avec certains membres dans la famille;

2. la fréquence des relations que la mère entretient avec ces membres (mesurée à partir du nombre de communications quotidiennes ou mensuelles: appels téléphoniques, visites, correspondance...);

3. la présence d'aide ou de services reçus par la répondante, selon les membres; et

4. le genre d'aide ou de services reçus (argent, soutien affectif, main-d'œuvre...) ainsi que leur fréquence.

Ces différents indicateurs ont été choisis spécifiquement parce qu'ils avaient été retenus dans des recherches antérieures. Les données obtenues ont toutes fait l'objet de traitements statistiques appropriés ${ }^{6}$.

Encore là, les résultats de l'enquête ne nous ont pas permis d'établir une différence qui serait liée à la langue, ni en ce qui a trait aux relations et aux communications entre les membres des familles anglophones et celles des familles francophones, respectivement, ni en ce qui concerne le genre d'aide ou de services échangés, ou encore en ce qui a trait au(x) membre(s) privilégié(s). La seule différence qui est apparue manifeste est l'étendue du 
"La seule différence qui est apparue manifeste est l'étendue $d u$ réseau familial auquel on fait référence : les francophones ont souvent mentionné plus du double de "personnes apparentées» que les anglophones;..." "...la famille francoontarienne se distingue peu, dans les faits, de la famille anglophone...» réseau familial auquel on fait référence: les francophones ont souvent mentionné plus du double de «personnes apparentées» que les anglophones; ceci confirme, si besoin était, qu'effectivement, dans les deux générations précédentes, la famille francophone était hors de tout doute plus nombreuse. Mais avoir plus de "parents» n'implique pas nécessairement avoir plus de relations avec ces nombreux parents ${ }^{7}$.

\section{Conclusion du deuxième volet}

Nous avons expliqué les résultats du peu de différences entre les deux groupes en développant une réflexion sur le paradoxe vécu par les Franco-Ontariennes prises entre, d'un côté, les paramètres de leur historicité de minoritaire et, de l'autre, comme tout individu actuel, le nouveau sens social que prend la famille dans la société postmoderne, c'est-à-dire son individualisation et les conséquences qui en résultent sur les structures sociales de référence dans l'identité de l'individu.

À cela s'ajoute un autre aspect des sociétés postmodernes qu'il convient de souligner parce qu'il a une incidence certaine sur les définitions du contexte social et sur les comportements dans leur ensemble. Dans des sociétés de masse-médiatisation, on assiste à une certaine homogénéisation des populations quant à leur vision du monde, leurs comportements, leurs désirs et souvent même leurs symboles comme l'ont démontré certains travaux récents (Laflamme et Dennie 1990; Laflamme et Berger 1988). Pourquoi la population francophone du Nord de l'Ontario ferait-elle exception à la règle? Ne vit-elle pas dans un contexte présentant plusieurs similitudes avec celui dans lequel vit aussi la population anglophone qu'elle côtoie? Mêmes organisations familiales, mêmes horaires, mêmes soucis, mêmes aspirations de confort et de réussite et, souvent, même accès aux médias anglophones.

Ainsi, tant dans la configuration du profil statistique que dans une comparaison des solidarités familiales, la famille francoontarienne se distingue peu, dans les faits, de la famille anglophone en Ontario. 


\section{Ethos familial et contexte minoritaire ${ }^{8}$}

«...la famille se produit dans une culture; comme elle et par son inscription en elle, elle est aussi le résultat d'un contexte et d'une histoire, mais elle est plus que la seule manifestation de la culture...»
Pourtant, des différences entre elles existent sûrement, ne seraitce que, comme cela a été mentionné, parce qu'il y en a une trace sociale : sur le plan affectif, les Franco-Ontariens et les FrancoOntariennes y croient; socialement, ils et elles se reconnaissent une spécificité en leur famille, leurs constructions symboliques, leur idée de leur famille postule cette différence. Il y aurait donc là une sorte d'ethos familial particulier qu'il nous appartient de découvrir. J'entends par ethos familial un état émotionnel, une vision, une représentation de la famille, un sens affectif convergeant, pourrait-on dire, tissé dans la trame du vécu quotidien de l'individu et cristallisé en une certitude nourrie à même la socialisation, développant un sentiment puissant d'appartenance à un groupe apparenté, une conviction de son identité familiale. On peut parler d'ethos familial spécifique lorsque cette vision, ce sentiment se retrouvent, dans les mêmes termes — c'est-à-dire s'exprimant à travers des énoncés plus ou moins logiquement structurés entre eux, et donc repérables par l'analyse - chez les individus d'un même groupe.

On aurait certes pu, plutôt, se référer à une terminologie renvoyant à une culture familiale; mais il m'apparait qu'aborder la question par l'angle de l'«ethos» permet de partir des individus eux-mêmes et d'insister davantage sur la dimension affective des liens entre famille et contexte socio-historique, contrairement aux analyses habituelles où l'on tente, de l'extérieur, de cerner les rapports de reproduction du groupe minoritaire en faisant état des fonctions des diverses institutions - dont la famille. Certes, la famille se produit dans une culture; comme elle et par son inscription en elle, elle est aussi le résultat d'un contexte et d'une histoire, mais elle est plus que la seule manifestation de la culture, ne serait-ce que parce que les individus qui y évoluent produisent leur propre dynamique de relations et d'émotions au delà des représentations symboliques de la culture. C'est cet ensemble d'éléments construisant l'ethos spécifique de la famille francoontarienne que la troisième étape de la recherche a voulu explorer. 


\section{L'enquête}

La collecte de données s'est faite à partir d'entrevues réalisées auprès de quinze mères francophones (ou bilingues) et de quinze mères anglophones, aux mois de mai et juin 1996, dans la région de Sudbury. L'échantillon a été construit en grande partie de répondantes provenant de la population des personnes ayant participé à l'étape II de la recherche.

Les entrevues se sont déroulées à partir d'un questionnaire semi-structuré, comprenant sept questions d'opinion, et construit dans les deux langues. On interrogeait les participantes sur leur vision de la famille, leur famille d'origine, leur famille actuelle et leurs définitions de la famille anglo-ontarienne et francoontarienne. Par la suite, on leur demandait d'établir une comparaison entre leur famille et des familles de leur entourage et, en outre, d'évaluer dans quelle mesure elles sont typiques comme famille anglo-ontarienne ou franco-ontarienne, selon le cas.

Les verbatims des entrevues ont été soumis, dans un premier temps, à une analyse de contenu classique, de type thématique. Après avoir identifié les grandes catégories thématiques, en fonction des questions posées, chaque catégorie a été subdivisée en autant de sous-catégories que l'exigeaient les énoncés exprimés sur le thème. Puis ces énoncés ont été regroupés selon la méthode des similitudes et des oppositions. L'énoncé servait ainsi d'unité de sens, une phrase pouvant évidemment comprendre plusieurs énoncés.

De cette manière, il a été possible d'identifier, de façon proportionnelle, l'intérêt et l'intensité que chaque personne interviewée (et, par la suite, chaque groupe) consacre à chacun des thèmes, de relever la variation et la complexité des idées exprimées ainsi que les différentes contradictions impliquées dans les réflexions présentées. C'est à ce niveau que s'est construite la comparaison entre les deux groupes. Ainsi au delà d'une seule analyse basée sur les fréquences, il s'agissait, en quelque sorte, d'une interprétation à saveur plus complexe, une forme d'herméneutique. Chaque verbatim a été codifié et analysé dans sa langue, c'est donc dire qu'il n'y a pas eu de traduction des entrevues de langue anglaise. 
"L'analyse des données révèle plusieurs différences dans les construits symboliques des personnes de chacun des deux groupes linguistiques..."
L'analyse des données révèle plusieurs différences dans les construits symboliques des personnes de chacun des deux groupes linguistiques lorsqu'elles s'expriment sur le sujet, dont on présente ici certains éléments?.

\section{Les francophones s'expriment plus sur le sujet}

Une première distinction frappante, entre les deux groupes linguistiques est que les francophones s'expriment plus sur le sujet que les anglophones; elles «en ont plus à dire». On trouve, en effet, une fois et demie plus d'énoncés chez les mères francophones, quel que soit l'aspect du sujet invoqué. C'est une problématique qui, tout au long de la lecture des entrevues, apparait chez elles plus réfléchie, plus pensée; on ressent nettement que c'est une préoccupation constante dans le groupe francophone.

\section{Le concept de famille}

Un deuxième élément de distinction apparait lorsque l'on tente de cerner la notion de famille. En effet, alors que, en ce qui a trait au concept général de famille, autant les francophones que les anglophones s'y réferent majoritairement encore selon la structure moderne de la famille nucléaire: père, mère, enfant-s — et ce, même si plusieurs des personnes interviewées vivent des situations familiales de mère seule - , dès que l'on aborde la notion de famille immédiate, il y a divergence entre les deux groupes. En effet, pour les francophones de l'échantillon, la notion de famille immédiate a d'entrée de jeu une acception plus large, plus vaste que chez les anglophones : la plupart du temps y sont inclus, en plus des enfants et du conjoint (lorsque c'est le cas), des membres de la famille d'origine, tantôt les parents, tantôt les soeurs ou les frères; par contre, chez les mères anglophones, la famille immédiate est presque exclusivement composée des membres de la stricte famille de procréation.Ainsi, on peut déjà constater que les francophones auront, dans l'ensemble, une représentation plus large de la famille comprenant leur famille immédiate (famille actuelle + famille d'origine) et la famille étendue, contrairement aux anglophones pour qui la famille actuelle et la famille d'origine sont nettement distinctes et qui font peu référence à leur famille étendue. 
"...il y a là un

amalgame certain entre rapport à l'ethnie et rapport à la famille.»

\section{La famille franco-ontarienne vue par ses membres}

Les résultats ont aussi permis de confirmer que les représentations des Franco-Ontariennes à propos de leur famille se construisent à partir des éléments déjà identifiés et que nous avons présentés au début comme les énoncés présents dans la rumeur sociale: très forte intensité des liens familiaux; haute fréquence des rencontres et des regroupements de tous types accompagnés d'un grand plaisir à être ensemble, d'un certain sens de la fête et d'une insistance sur les traditions. À cela s'ajoutent l'importance accordée à la valorisation du fait français et l'effort quotidien constant nécessité par le maintien de la langue française et de la culture francoontarienne au sein du foyer. Tous ces éléments, redits sans cesse par les mères francophones tout au long des entrevues, révèlent à quel point le rapport à la famille, chez elles, est définitivement lié à une question identitaire: il y a là un amalgame certain entre rapport à l'ethnie et rapport à la famille. ${ }^{10}$

\section{La famille franco-ontarienne vue par les mères anglophones}

Étonnamment, lorsque certaines des mères anglophones s'expriment sur la famille franco-ontarienne, elles présentent des opinions qui reprennent en partie les éléments soulevés par les francophones, particulièrement en ce qui touche aux liens familiaux et à la tradition. À cette différence près cependant: ces éléments ne se parent pas toujours de la même connotation; parfois survalorisés, parfois lieux d'ambiguité, les traits mentionnés donnent, en effet, souvent lieu à l'expression d'un paradoxe. Rarement présentés comme totalement positifs ou totalement négatifs, ils expriment une certaine dévalorisation de l'Autre en même temps qu'ils manifestent une certaine aspiration, une forme d'envie, un désir de devoir-être, parfois même présenté comme idéal-typique, de ce que pourraient être les «vraies valeurs familiales, ou «une vraie famille».

\section{La famille anglophone vue par ses membres}

Par contre, les mères anglophones ont peu à dire sur la famille anglo-ontarienne : c'est une famille où l'on parle anglais, elle n'a rien de spécial, elle est comme les autres familles, répéteront-elles. 
De toute évidence, dans son aspect socio-linguistique, la famille ne constitue pas une problématique du côté anglophone. On n'y pense pas, on ne s'en préoccupe pas, semble-t-il.

N'étant soumise à aucun danger d'assimilation, sentant sa situation très stable, dominante même, rien dans les représentations qui sont socialement véhiculées à son égard, par ses membres, ne renvoie à une obligation de se positionner, de se démarquer, de se reconnaître une différence, de trouver des caractéristiques sur lesquelles elle pourrait fonder sa spécificité.

\section{La famille anglo-ontarienne vue par les mères francophones}

Les mères francophones, de leur côté, sont aussi volubiles en ce qui a trait à la famille anglo-ontarienne qu'elles l'ont été en ce qui concerne leur propre famille. Mais bien peu d'énoncés comportent des éléments positifs. Deux trames de fond servent de fil directeur aux opinions exprimées: d'un côté, on présentera la famille anglophone comme plus froide, moins accueillante, qui sait moins s'amuser; de l'autre côté, c'est sa position dominante, son invulnérabilité qui est soulevée et qui renvoie au havre que doit représenter un lieu, un contexte familial où l'on n'est pas tenue d'être constamment vigilante, où la sauvegarde de la langue n'est pas un enjeu des relations familiales.

\section{Conclusion du troisième volet : la construction d'un ethos}

On a pu constater, dans le discours des mères présentés dans le troisième volet de cette recherche, que parler de la famille francoontarienne, c'est produire une adéquation entre rapport à la famille et rapports à l'ethnie à travers le rapport à la langue et à la culture. En effet, alors que dans le discours des mères anglophones on parle peu de traditions familiales et lorsque l'on en parle, on ne les identifie pas comme traditions culturelles (ou ethniques) - on parlerait plutôt d'habitudes ou de rites —, dans le discours des mères franco-ontariennes, cela semble indissociable. Et même plus: on ne fait pas que parler des traditions, on les vante, on les «emphatise», si l'on peut dire, on les idéalise. De la même manière, on ne fait pas que parler du type ou de la fréquence des rapports entre les membres, on les loue, on les magnifie, on les surqualifie à la limite. 
"On assiste donc à une sorte de survalorisation des attributs de la famille franco-ontarienne...»
«En fait, c'est sa production en contexte minoritaire qui lui donne sa couleur locale, qui construit l'idée de sa spécificité, l'obligation d'en développer la conviction.»
On assiste donc à une sorte de survalorisation des attributs de la famille franco-ontarienne, survalorisation qui ne peut se concevoir sans une dévalorisation de l'Autre, sans un dénigrement de la famille anglophone. Cette représentation polarisée des familles anglo-ontarienne et franco-ontarienne renvoie bien à l'analyse du paradoxe identitaire en contexte minoritaire, telle qu'elle a déjà été développée ailleurs (Laflamme et Dennie 1990).

On retrouve même certains aspects de cette valorisation dans le discours des anglophones. Mais cette reconnaissance des aspects positifs de la famille franco-ontarienne par les anglophones se fait sur un fond d'historicité bien particulière: on éprouve peu de crainte, en tant que groupe majoritaire, à reconnaitre certaines qualités aux minoritaires, à les survaloriser même, tant que cela reste du domaine du privé, de l'ordre du restreint, du distinct... mais on ne saurait avoir la même attitude lorsque les francophones sortent de cet espace bien délimité. La survalorisation ici répond à une fonction différente:le maintien de la mise à distance de l'Autre, dans la reproduction du statu quo des rapports de pouvoir.

C'est ce fond d'historicité qui donne à la famille francoontarienne un ethos spécifique. Parce que ce n'est pas tant qu'elle soit si différente des autres familles, mais l'obligation ajoutée (comme on dirait la valeur ajoutée) de la valorisation du fait français et l'effort quotidien nécessité par le maintien de la langue, au sein du foyer, colorent ses rapports à la culture et aux rencontres familiales. En fait, c'est sa production en contexte minoritaire qui lui donne sa couleur locale, qui construit l'idée de sa spécificité, l'obligation d'en développer la conviction.

Car, ainsi que l'avait révélée la première étape de cette recherche, peu d'éléments dans les indicateurs objectifs permettent de croire à une réelle différence entre familles franco-ontarienne et anglo-ontarienne. De même, comme, encore là, nous l'avons démontré lors de la deuxième étape du processus, il n'est pas vrai de croire, malgré ce qu'en disent les Franco-Ontariens et les Franco-Ontariennes - et même certaines mères anglophones comme le montre cette recherche - que les communications sont plus fréquentes dans leurs familles, et qu'ils et elles établissent 
«...dis-le moi en

français, s'il te

plaît...” plus de réseaux d'échange et de soutien à partir de leurs familles que les membres de la famille anglo-ontarienne.

Mais, dans ce sentiment de vivre en perpétuel sursis; dans l'urgence du danger côtoyé tous les jours, dans l'effort, la lutte, le conflit aussi parfois, la fatigue souvent, de devoir faire plus tout le temps; dans l'obligation de ne pas baisser les bras et d'assumer sa conviction en répétant quotidiennement à ses enfants «dis-le moi en français, s'il te plaît» — développant à travers la difficulté, le plaisir toujours renouvelé d'être ensemble, là où l'on peut vivre «toute en français» - ...c'est là que se construit l'ethos de la famille franco-ontarienne, l'ethos qui pare les activités familiales, les traditions, les rites, les rencontres d'une aura qui ne se comptabilise pas, qui ne répond pas à des indicateurs objectifs, mais qui crée les principes générateurs de prises de position nécessaires à sa reproduction comme famille francophone et à sa présence, comme mythe, dans l'imaginaire collectif ontarien.

\section{Conclusion}

Il va sans dire que les conclusions auxquelles nous convient les résultats présentés, notamment celles issues de la troisième phase de la recherche, mériteraient d'être confrontées à des analyses reprenant les mêmes hypothèses, mais portant sur d'autres groupes sociaux, comme les pères franco-ontariens ou les mères francophones vivant ailleurs qu'au Nord de l'Ontario, ou encore sur des échantillons stratifiés par groupe d'âge. Malgré l'intérêt que ces conclusions comportent, il serait hasardeux, en effet, de généraliser à l'ensemble de la population franco-ontarienne, des résultats obtenus sur un échantillon aussi restreint.

Mais, au delà de ces précisions méthodologiques, les éléments qui ont été mis à jour au cours des trois volets de cette recherche sur la famille franco-ontarienne nous apparaissent importants. Non seulement nous obligent-ils, à l'aide de la comparaison établie avec la famille anglo-ontarienne, à relativiser nos croyances sur la 
famille francophone, mais surtout ils nous permettent d'appréhender un aspect du vécu des francophones en milieu minoritaire à travers leurs représentations de la famille. Entre mythe et réalité, la conviction sur laquelle repose la spécificité de la famille francoontarienne est d'un intérêt non négligeable tant pour l'étude des représentations sociales que pour les recherches sur les francophones en milieu minoritaire.

\section{Bibliographie}

ASSOCIATION CANADIENNE-FRANÇAISE DE L'ONTARIO (1988). Les francophones tels qu'ils sont en 1986, Recueil Statistique, Vanier, ACFO.

BAWIN-LEGROS, B. (1988). «Fonctions et structures familiales. Théorie, modèles et typologies», dans Familles, mariage, divorce: une sociologie des comportements familiaux contemporains, Bruxelles, Pierre Mardaga, chap. 1.

BEAUVOLSK, M-A. (1995). «Le phénomène d'ajustement de la famille reconstituée francoontarienne», dans C. Bernier, S. Larocque et M. Aumond (dir. de publ.), Familles francophones: multiples réalités, Actes du colloque, Sudbury, Institut franco-ontarien, 181-194.

BERNARD, R. (dir.) (1990). Vision d'avenir, Ottawa, Fédération des jeunes Canadiens-Français, Livre I : Le déclin d'une culture, Livre II : Le choc des nombres, Ottawa, Fédération des jeunes CanadiensFrançais.

BERNIER, C. (1995). «Familles franco-ontariennes : un profil statistique» dans C. Bernier, S. Larocque et M.Aumond (dir. de publ.), Familles francophones: multiples réalités, Actes du colloque, Sudbury, Institut franco-ontarien, 107-134.

BERNIER, C. (1994). «Une analyse sociologique féministe est-elle possible? Analyse critique autour des Actes du colloque Les femmes francophones en milieu minoritaire», Revue du Nouvel Ontario, no $15,119-156$.

BERNIER, C. et C.VAILLANCOURT (1996). «Solidarité familiale et différentiation selon la langue: la fin d'un mythe?», Revue du Nouvel Ontario, no 19, 59-87.

BLISHEN, B., W. CARROLL et C. MOORE (1987). «The 1981 Socioeconomic Index for Occupations in Canada", Revue canadienne de sociologie et d'anthropologie, vol. 24, 465-488.

BRETON, R. (1983). «La communauté ethnique, communauté politique», Sociologie et Société, vol. 15, no 2, 23-38.

CARDINAL, L. et C. CODERRE (1992). «Les francophones telles qu'elles sont : les Ontaroises et l'économie», Revue du Nouvel Ontario, no 12,151-181.

CARRIÈRE, F. (1993). «La métamorphose de la communauté franco-ontarienne», dans C.J.Jaenen (dir. de publ.), Les Franco-Ontariens, Ottawa, Presses de l'Université d'Ottawa, 305-340.

CHOQUETTE, R. (1977). Langue et religion: histoire des conflits anglo-français en Ontario, Ottawa, Éditions de l'Université d'Ottawa.

CONNIDIS, I. A. (1989). Family Ties and Aging, Toronto, Butterworths. 
DANDURAND, R. (1995). "Une théorie qui se cherche. Pistes de réflexion pour une analyse sociale de la vie familiale», dans C. Bernier, S. Larocque et M. Aumond (dir. de publ.) Familles francophones: multiples réalités, Actes du colloque, Sudbury, Institut franco-ontarien, 19-34.

DANDURAND, R. (1987). Couple et parents des années quatre-vingts, Québec, IQRC.

DOISE,W. (1986). «Les représentations sociales : définition d'un concept» dans W. Doise et A. Palmonari (dir. de publ.), L'étude des représentations sociales, Paris, Delachaux et Niestlé, 81-94.

DOISE, W., A. CLEMENCE et F. LORENZI-CIOLDI (1992). Représentations sociales et analyses de données, Grenoble, Presses Universitaires de Grenoble.

GERVAIS, G. (1993). «L'Ontario français (1821-1910)», dans C.J. Jaenen (dir. de publ.), Les FrancoOntariens, Ottawa, Presses de l'Université d'Ottawa, 49-125.

HELLER, M. et L. LEVY (1993a). «Des femmes franco-ontariennes en situation de mariage mixte : vivre sur une frontière linguistique», dans L. Cardinal (dir. de publ.), Une langue qui pense, Ottawa, Presses de l'Université d'Ottawa, 11-27.

HELLER, M. et L. LEVY (1993b). «La femme franco-ontarienne en situation de mariage mixte: désirs et réalités dans l'éducation des enfants» dans Les femmes francophones en milieu minoritaire: état de la recherche. Actes du colloque, Sudbury, Institut Franco-Ontarien.

HELLER, M. et L. LEVY (1992). «La femme franco-ontarienne en situation de mariage mixte», Recherches féministes, vol. 5, no 1, 59-82.

HERRY, Y. et D. LÉVESQUE (1994). «Les caractéristiques des adolescents», dans Éducation Canada, vol. 34, no 3, 21-33.

JUTEAU, D. et L. SÉGUIN-KIMPTON (1993). «La collectivité franco-ontarienne : structuration d'un espace symbolique et politique», dans C. J. Jaenen (dir. de publ.), Les Franco-Ontariens, Ottawa, Presses de l'Université d'Ottawa, 265-304.

KELLERHALS,J., L.VALENTE, P.Y.TROUTOT et E. LAZEGA (1984). Microsociologie de la famille, Paris, Presses Universitaires de France.

KULIS, S. S. (1991). Why Honor Thy Father and Mother? Class, Mobility and Family Ties in Later Life, New York, Garland Publishing.

LACOURSE, M.T. (1994). Famille et société, Québec, McGraw-Hill.

LAFLAMME, S. et J. BER GER (1988). «Compétence linguistique et environnement social», The Canadian Modern Language Review/La Revue canadienne des langues vivantes, vol. 44, no 4, 619-638.

LAFLAMME, S. et D. DENNIE (1990). L'ambition démesurée, Sudbury, Institut franco-ontarien et Prise de Parole.

LEE, T. R., J. A. MANCINI et J. W. MAXWELL (1990). «Sibling Relationships in Adulthood: Contact Patterns and Motivations", Journal of Marriage and the Family, vol. 52, 431-440.

LITWAK, E. (1965). «Extended Kin Relations in an Industrial Democratic Society», Social structure and the Family: Generational Relations, Toronto, Prentice Hall Inc., 290-323.

MARTEL, M. (1993). «De la certitude au doute: l'identité canadienne française de l'Ontario de 1937 à 1967» dans L. Cardinal (dir. de publ.), Une langue qui pense, Ottawa, Presses de l'Université d'Ottawa, 65-76.

MCKEE-ALLAIN, I. (1995). "Les familles acadiennes des années 1990: profil et enjeux.» dans C. Bernier, S. Larocque et M.Aumond (dir. de publ.), Familles francophones: multiples réalités, Actes du colloque, Sudbury, Institut franco-ontarien, 93-106. 
MCKEE-ALLAIN, I. (1989). «Les productrices d'ethnicité en Acadie : perspectives théoriques», Égalité, vol. 24, 45-68.

MICHEL, A. (1972). Sociologie de la famille et du mariage, Paris, Presses Universitaires de France.

MUTRAN, E. (1985). «Intergenerational Family Support Among Blacks and Whites: Response to Culture or to Socioeconomic Differences", Journal of Gerontology, vol. 40, 382-389.

PARSONS,T. et R. BALES. (1955). Family: Socialization and Interaction Process, NewYork, Free Press, 3-33. PITROU, A. (1994). Les politiques familiales : approches sociologiques, Paris, Syros.

PITROU, A. (1992). Les solidarités familiales : vivre sans famille?, Toulouse, Privat.

ROBERGE, P. (1994). «Difficultés de communication dans les familles recomposées francophones du Nord-est de l'Ontario", Revue du Nouvel Ontario, no 15, 35-50.

SÉGALEN, M. (1981). Sociologie de la famille, Paris, Armand Colin.

SINGLY, F. de (dir.) (1991). La famille, l'état des savoirs, Paris, Éditions La découverte.

SMITH, T. A. (1991). "Family Cohesion in Remarried Families», Journal of Divorce and Remarriage, vol. 17, 49-66.

SPITZE, G. et J. R. LOGAN (1991). «Sibling Structure and Intergenerational Relations», Journal of Marriage and the Family, vol. 53, 871-884.

STATISTICS CANADA. (1981). Standard Occupational Classification (1980), Ottawa, Ministère des Approvisionnements et services.

STATISTIQUE CANADA (1994) La famille et les amis. Enquête sociale générale, Ottawa, Ministre de l'industrie, des Sciences et de la Technologie. Catalogue: 11-612F, no 9.

\section{Notes}

1. D'autres recherches, comme celles de M. Heller et L. Lévy (1992,1993a, 1993b) ont porté sur les liens entre ces trois problématiques, mais il s'agissait d'études sur les familles «bilingues», c'est-à-dire issues de mariages mixtes et donc non expressément francophones.

2. Voir à ce sujet l'article de Bernier (1995).

3. Pour les données complètes, les valeurs statistiques et l'analyse détaillée, voir l'article de Bernier (1995).

4. Voir à ce sujet l'article de Bernier et Vaillancourt (1996).

5. L'idée d'interviewer les mères a été retenue parce que ce sont encore les femmes qui entretiennent le plus les liens familiaux et que ce sont elles qui sont le plus souvent parent seul.

6. Pour les données complètes, les valeurs statistiques et l'analyse détaillée, voir l'article de Bernier et Vaillancourt (1996).

7. Pensons ici à la définition de parentèle, mise de l'avant par Martine Ségalen, pour expliquer le «choix» que, en famille postmoderne, les personnes font de celles avec qui elles auront des relations suivies: «La parentèle, cette constellation souple de parents 
avec laquelle on peut choisir d'avoir ou de ne pas avoir de relations, [qui] détermine des réseaux qui unissent entre eux les divers groupes domestiques apparentés» (1981:81).

8. Voir aussi l'article de Bernier, C. «Ethos familial et contexte minoritaire: comparaison des représentations de la famille entre mères francophones et anglophones de la région de Sudbury", à paraitre dans de la Revue du Nouvel-Ontario.

9. Pour la présentation des différents énoncés retenus dans l'analyse et une interprétation détaillée des résultats, voir l'article de Bernier à la Note 8 .

10. Comme nous l'avons expliqué antérieurement «...de totalement immergée, coulée pourrait-on dire, qu'elle était dans la communauté ethnique ou politique jadis, la famille, en société postmoderne, prendrait sa propre place distincte, dégagée de la communauté, ce qui aurait pour effet de transformer les liens identitaires de l'individu à ces deux instances: c'est médiatisé par la famille qu'on s'identifiait à la communauté, jadis; c'est en rapport à ses propres valeurs, priorités, croyances, de façon individuelle, que l'on s'y identifie aujourd'hui. Ce dédoublement de l'axe du référent identitaire produit, semble-t-il, en contexte minoritaire, un décalage important: alors que, comme toutes les autres familles, la famille franco-ontarienne subit les variations propres aux familles postmodernes et au contexte global, les Franco-Ontariens et les FrancoOntariennes voient encore, dans leurs référents symboliques, leur famille comme intégrée à la communauté ethno-politique; d'où confusion, dans les représentations, entre sentiment d'appartenance à l'ethnie (identité nationalitaire) et sens d'appartenance à sa famille» (Bernier et Vaillancourt 1996: 80). 